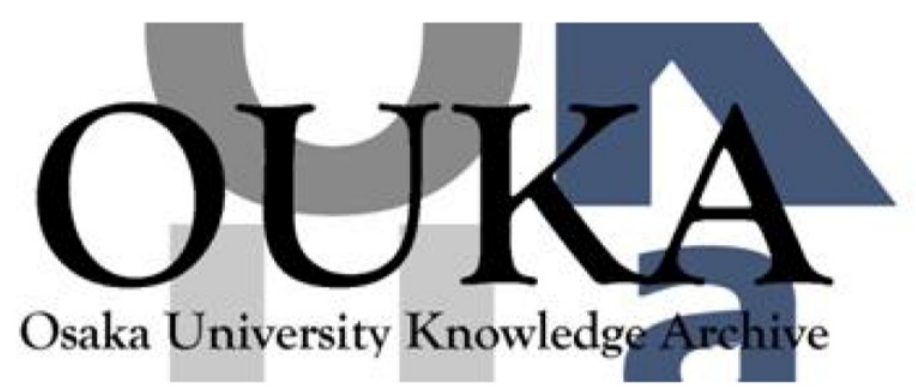

\begin{tabular}{|c|c|}
\hline Title & $\begin{array}{l}\text { Ion acceleration from the shock front induced } \\
\text { by hole boring in ultraintense laser-plasma } \\
\text { interactions }\end{array}$ \\
\hline Author (s) & Habara, H.; Lancaster, K. L. ; Karsch, S. et al. \\
\hline Citation & Physical Review E. 70(4) p. 046414 \\
\hline Issue Date & $2004-10$ \\
\hline oaire:version & VoR \\
\hline URL & https://hdl. handle. net/11094/3465 \\
\hline rights & $\begin{array}{l}\text { Habara, H., Lancaster, K. L., Karsch, S., } \\
\text { Murphy, C.' D., Norreys, P. A. , Evans, R.'G., } \\
\text { Borghesi, M.,' Romagnani, L. 'Zepf, M., } \\
\text { Norimatsu, T. , Toyama, Y., Kodama, R., King, J. } \\
\text { A., Snavely, R., AkLi, K., Zhang, B., Freeman, } \\
\text { R., Hatchett, S., Mackinnon, A. J., Patel, P., } \\
\text { Key, M. H., Stoeckl, C. Stephens, R. B., } \\
\text { Fonseca, R. A..'Silva, L. O, Physical Review } \\
\text { E, 70, 4, 046414, 2004-10. "Copyright } 2004 \text { by } \\
\text { the American Physical Society." }\end{array}$ \\
\hline Note & \\
\hline
\end{tabular}

Osaka University Knowledge Archive : OUKA

https://ir. Library. osaka-u. ac. jp/

Osaka University 


\title{
Ion acceleration from the shock front induced by hole boring in ultraintense laser-plasma interactions
}

\author{
H. Habara, K. L. Lancaster, S. Karsch, C. D. Murphy, and P. A. Norreys \\ Central Laser Facility, Rutherford Appleton Laboratory, Chilton, Didcot, Oxon OX11 OQX, United Kingdom \\ R. G. Evans \\ Blackett Laboratory, Imperial College, Prince Consort Road, London SW7 2BZ, United Kingdom \\ M. Borghesi, L. Romagnani, and M. Zepf \\ Department of Pure and Applied Physics, Queens University of Belfast, Belfast BT7 1NN, United Kingdom \\ T. Norimatsu, Y. Toyama, and R. Kodama \\ Institute of Laser Engineering, Osaka University, Suita, 565-0871 Osaka, Japan \\ J. A. King, R. Snavely, K. Akli, B. Zhang, and R. Freeman \\ Department of Applied Science, University of California, Davis, 1 Shields Avenue, Davis, California 95616-8254, USA \\ S. Hatchett, A. J. MacKinnon, P. Patel, and M. H. Key \\ Lawrence Livermore National Laboratory, P. O. Box 808, Livermore, California 94550, USA \\ C. Stoeckl \\ Laboratory for Laser Energetics, University of Rochester, Rochester, New York 14623, USA \\ R. B. Stephens \\ General Atomics, P. O. Box 85608, San Diego, California 92186-5608, USA \\ R. A. Fonseca and L. O. Silva \\ GOLP/Centro de Fisica dos Plasmas, Insituto Superior Tecnico, 1049-001 Lisbon, Portugal \\ (Received 6 November 2003; revised manuscript received 1 June 2004; published 29 October 2004)
}

\begin{abstract}
Ion-acceleration processes have been studied in ultraintense laser plasma interactions for normal incidence irradiation of solid deuterated targets via neutron spectroscopy. The experimental neutron spectra strongly suggest that the ions are preferentially accelerated radially, rather than into the bulk of the material from three-dimensional Monte Carlo fitting of the neutron spectra. Although the laser system has a $10^{-7}$ contrast ratio, a two-dimensional magnetic hydrodynamics simulation shows that the laser pedestal generates a $10 \mu \mathrm{m}$ scale length in the coronal plasma with a $3 \mu \mathrm{m}$ scale-length plasma near the critical density. Two-dimensional particle-in-cell simulations, incorporating this realistic density profile, indicate that the acceleration of the ions is caused by a collisionless shock formation. This has implications for modeling energy transport in solid density plasmas as well as cone-focused fast ignition using the next generation PW lasers currently under construction.

DOI: 10.1103/PhysRevE.70.046414

PACS number(s): 52.35.Tc, 52.38.Kd
\end{abstract}

Ion-acceleration processes in high-power, ultraintense laser-plasma interactions are an extremely important area of research in the fast ignitor (FI) [1] approach to inertial fusion energy as a source of additional heating. These energetic particles are also expected to be useful in many applications, such as nuclear physics [2,3], astrophysics [4], and even in biological and medical application as a bright particle source.

In recent studies, energetic ions with energies of up to $60 \mathrm{MeV}$ have been reported in intense laser-plasma interactions from the rear side of the target $[5,6]$. Ion-acceleration mechanisms at the front surface of the solid target have been shown to be affected by both the plasma density scale length and the electron acceleration processes [7]. For example, when the intensity contrast ratio of pedestal to main pulse is
$10^{-7}$, charge separation acceleration dominates for irradiances up to $5 \times 10^{17} \mathrm{~W} \mathrm{~cm}^{-2} \mu \mathrm{m}^{2}$ [8], whereas hole-boring acceleration occurs with lower contrast ratios (a few $10^{-4}$ ) and somewhat higher irradiances $\left(5 \times 10^{19} \mathrm{~W} \mathrm{~cm}^{-2} \mu \mathrm{m}^{2}\right)$ [9]. In addition, recent calculations suggest that a collisionless shock acceleration process occurs for the high-intensity contrast ratio, ultrahigh intensity interactions (I $\approx 10^{20} \mathrm{~W} / \mathrm{cm}^{2}$ ) [10]. It is therefore important to understand the dependence on the scale length of preformed plasma for these processes, particularly for shorter scale lengths where the higher acceleration gradients can be expected.

Since these ions must be accelerated by the electric fields set up by the fast electrons around the focal region, measuring their dynamics can indicate the role that these fields play 

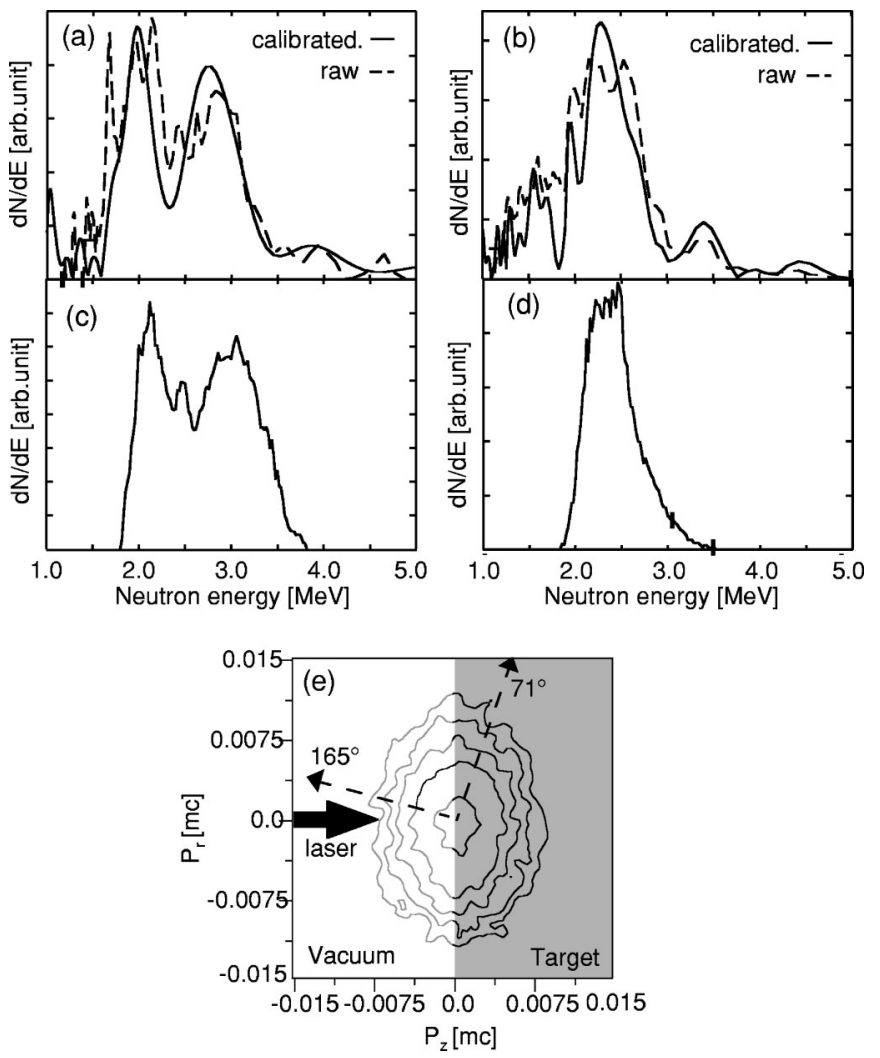

FIG. 1. Neutron spectra from experiment and Monte Carlo fitting. Experimental neutron spectrum detected at (a) $71^{\circ}$ and (b) $165^{\circ}$. The solid lines represent the unfolded spectrum using a response function of the detector. The dashed lines are raw data. (c) and (d) Best-fit calculated neutron spectra using a 3D Monte Carlo code assuming a Maxwellian distribution. (e) The best-fit ion momentum distribution.

in energy transport in solid density plasmas. For example, a ballooning effect-where the hot electron source size is always much larger than the focal spot dimensions-has been reported in a number of experiments recently [11,12]. For these reasons, we have studied the ion acceleration at the front surface of the target with a laser pulse that has an intensity contrast ratio of $10^{-7}$. We used spectroscopy on the neutrons generated from nuclear fusion reactions of accelerated ions via the beam-fusion process $[13,14]$ because this method can be used to derive the accelerated ion momentum distribution inside the overdense plasma, avoiding the influence of strong electric and magnetic fields around the target.

In this paper, the momentum distribution of accelerated ions is presented by unfolding the neutron spectra by numerical analysis using a three-dimensional (3D) Monte Carlo fitting procedure [15]. The deduced momentum distributions are compared with a two-dimensional particle-in-cell simulation using realistic plasma density profiles. They indicate that the acceleration of ions is caused by a collisionless shock resulting from the hole boring in the preformed plasma. We show that this effect must be taken into account when modeling present-day electron energy transport experiments in dense plasmas and has implications for efficient energy coupling to the dense core in fast ignition.
The experiment was performed at Rutherford Appleton Laboratory using the VULCAN laser facility [16]. This laser system produces pulses up to $90 \mathrm{~J}$ on target at a wavelength $1.05 \mu \mathrm{m}$ with a pulse duration of $0.9-1.2 \mathrm{ps}$. A deuterated plastic plane target was irradiated at normal incidence using an f/4 off-axis parabolic mirror. From the far-field pattern in an equivalent plane monitor and the $4 \mathrm{MeV}$ electron temperature (deduced from those electrons that escaped from the target into a spectrometer located at the chamber wall), the peak laser intensity was $5( \pm 3) \times 10^{19} \mathrm{~W} / \mathrm{cm}^{2}$ on target. The contrast ratio between the pedestal and the main pulse has been measured previously to be $10^{-7}: 1$ [17].

The neutron energy spectra were detected by a plastic scintillator combined with a photomultiplier tube through the time-of-flight (TOF) method. Two detectors were set at $71^{\circ}$ and $165^{\circ}$ from the target normal at $2.3 \mathrm{~m}$ and $1.9 \mathrm{~m}$ from the target. Each detector has $10 \mathrm{~cm}$ lead blocks in front as well as $5 \mathrm{~cm}$ blocks to the sides to reduce the gamma noise which would otherwise cause the detector to saturate. The energy resolution of the detector was about $200 \mathrm{keV}$ and the valid detectable range was under $15 \mathrm{MeV}$. The detection efficiency and the response function were calibrated from a thermal neutron signal generated from an implosion experiment using a deuterium-filled glass microballoon at Osaka University.

The momentum distribution of accelerated ions was evaluated by a 3D Monte Carlo calculation [15] to fit the calculation to the experimental spectra. The fitting was performed giving several momentum distributions, e.g., Maxwellian, monoenergetic, isotropic, anisotropic, etc.

Figure 1 shows the obtained neutron spectra at (a) $71^{\circ}$ and (b) $165^{\circ}$ from the laser incidence direction. The solid lines in the figures represent the unfolded spectra deconvolved with the response function of each detector, whereas the dashed lines indicate the raw spectra. The neutron yield per steradian in the forward and backward directions was $(2.81 \pm 0.20)$ $\times 10^{6}$ and $(2.65 \pm 0.19) \times 10^{6}$, respectively. The spectrum obtained from the detector located at the front of the target indicates a slight Doppler shift of the peak to the lowerenergy side of $2.45 \mathrm{MeV}$, indicating some ion acceleration into the bulk of the target with a lower-energy component. The neutron spectrum obtained from the detector located at theside of the target, on the other hand, showed the peak split at 2.1 and $2.8 \mathrm{MeV}$. This large Doppler upshift and downshift suggest the ions were accelerated with higher energies into the radial direction.

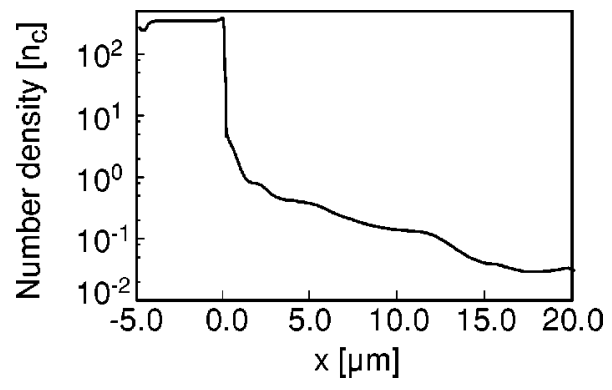

FIG. 2. Density gradient immediately before the main pulse irradiation calculated by POLLUX [18]. 


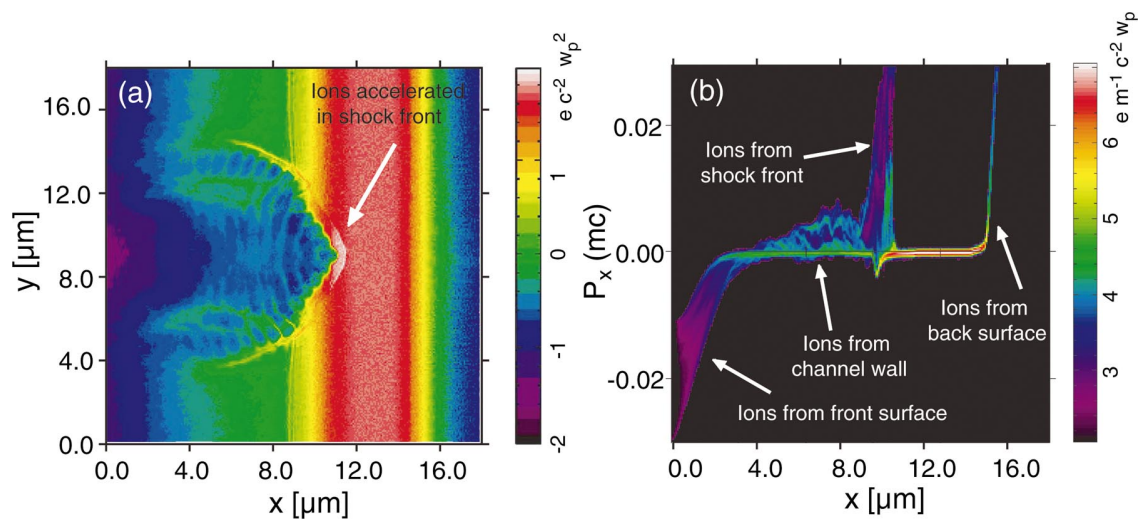

FIG. 3. (Color) Ion density and momentum-space diagram calculated by a 2D particle-in-cell simulation. (a) Density map from the main pulse irradiation at 300 fs. (b) Momentum-space diagram at $135 \mathrm{fs}$ for laser incidence direction.

Figures 1(c) and 1(d) show the best-fit spectra at $71^{\circ}$ and $165^{\circ}$ direction assuming the anisotropic ion momentum distribution as shown by the contour plot in Fig. 1(e). The gray and clear zones show the target and vacuum regions, respectively. The contour lines in the figure are plotted by every 2.5 relative ion number. The temperatures of the ion momentum are $40 \mathrm{keV}$ for those ions directed into the target and $80 \mathrm{keV}$ for the radial direction. The maximum ion energy was about $1 \mathrm{MeV}$ from the highest neutron energies. The neutron counts of the calculated spectra per steradian per ion are each $3.97 \times 10^{-8}$ for the $71^{\circ}$ and $3.04 \times 10^{-8}$ for the $165^{\circ}$ spectrum. This is similar to yields observed previously on VULCAN [13]. The total energy transferred to the deuterons was estimated from the experimental yield to be 0.7-4.2 J, corresponding to $1-5 \%$ of the incident laser energy.

The results indicate that the ions are accelerated preferentially in the radial direction. This indicates that the ion acceleration mechanism is not the static field induced by the charge separation [8], but is somewhat similar to the results from the "hole-boring model" for longer scale length interactions [9]. However, the contrast ratio of the VULCAN $100 \mathrm{TW}$ laser system is of order $10^{-7}$, therefore the scale length of the preformed plasma was expected to be short.

To check this expectation, the electron density gradient was calculated by a two-dimensional magnetic hydrodynamics (MHD) code, POLLUX [18], assuming that the preformed plasma is created only by the pedestal of the laser pulse and that the pedestal has a Gaussian shape with 300 ps pulse duration (full width at half maximum) from the temporal window of the optical switch in the laser system. The spatial profile of the pedestal is assumed to be the same as the main pulse. Figure 2 shows the density gradient for the target normal direction at $150 \mathrm{ps}$ from the beginning of the pedestal, just when the main pulse arrives on the target. The scale length at the critical density is $3 \mu \mathrm{m}$, but there is also a long underdense region following the steep gradient at the critical. The distance from the solid density surface to the $10^{20} / \mathrm{cm}^{3}$ contour is $10 \mu \mathrm{m}$, which agrees quite well with the results from shadowgram images taken earlier at the VULCAN facility [19].

Two-dimensional particle-in-cell (PIC) simulations were performed using the OSIRIS framework [20] to investigate the ion motion with an electron density gradient using the conditions calculated from the hydrocode. A fully ionized deuteron plasma was set at the right side from the center of the $512 \times 512$ cell simulation box $(18 \mu \mathrm{m}$ square $)$ and both boundaries were periodic. The $1 \mu \mathrm{m}$ laser light is incident onto the plasma slab from left to right with a $10 \mu \mathrm{m}$ spot size at an intensity of $8 \times 10^{19} \mathrm{~W} / \mathrm{cm}^{2}\left(a_{0}=8\right)$. The density gradient has a $3 \mu \mathrm{m}$ scale length at the critical density following an $8 \mu \mathrm{m}$ length underdense plasma to $10^{20} \mathrm{~cm}^{-3}$. The maximum density is limited to $30 \times$ critical for numerical stability reasons.

Figure 3(a) indicates the density space of the accelerated ions. The oblique collisionless shock is observed near the critical density in front of the hole-boring surface [21]. At the front of the shock wave, the ion density rises over the critical density and, in particular, reaches about $10^{23} \mathrm{~cm}^{-3}$ near the focal point. The ions are accelerated by the reflection at the density modulation (at $x=10.0 \mu \mathrm{m}$ ) as shown in Fig. 3(b),
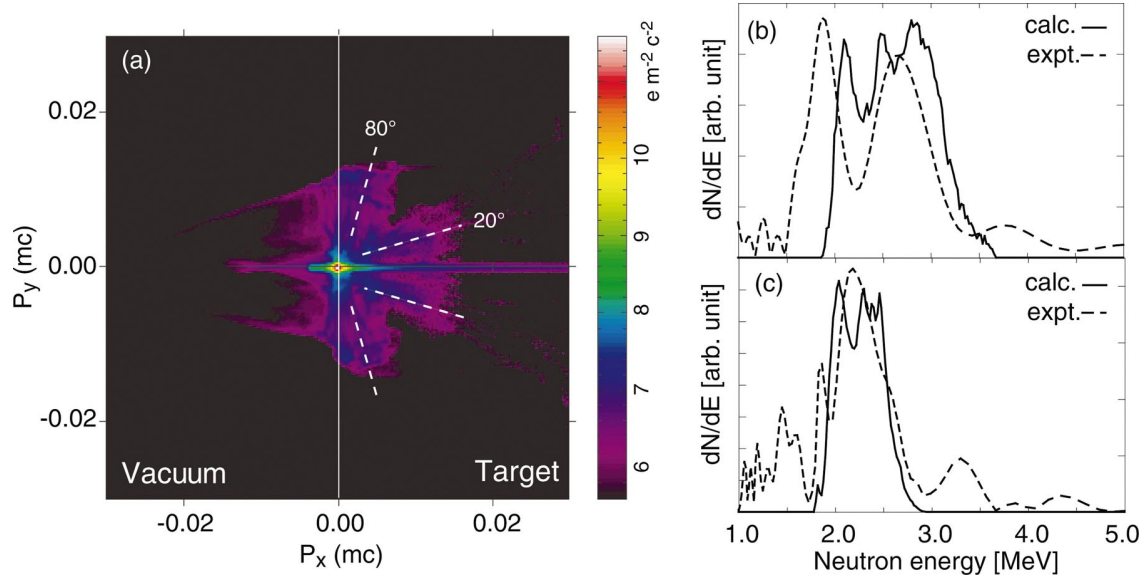

FIG. 4. (Color) (a) Ion momentum distribution by PIC calculation at $135 \mathrm{fs}$ from the beginning of the interaction averaged over the entire phase volume. (b) and (c) Neutron spectra calculated from the distribution (solid line) using the Monte Carlo code and the experimental result (dashed line). 
which shows a plot of the ion momentum at each position on the laser incidence axis. From $x=4.0$ to $9.0 \mu \mathrm{m}$, low-energy ions are accelerated into the target and are associated with the recession of the hole-boring surface in the underdense plasma. The narrow jet at $x=15 \mu \mathrm{m}$ is associated with the ions accelerated from the rear surface of the target $[22,23]$.

The ion momentum distribution is shown in Fig. 4(a). The well-collimated ions accelerated along $P_{y}=0$ correspond to the ions from the rear surface. On the other hand, ions are accelerated at two distinct angles of $20^{\circ}$ and $80^{\circ}$ from the laser incidence axis with both Maxwellian temperatures of approximately $180 \mathrm{keV}$. These directions correspond to the two angles of the shock front as shown in Fig. 3(a). Using this ion distribution, the neutron spectra at angles corresponding to those of the detectors were calculated using the Monte Carlo code as shown by the solid lines in Figs. 4(b) and 4(c) [the dashed lines in the figures are the experimental spectra already shown in Figs. 1(a) and 1(b)]. It is clear that the calculated spectra agree reasonably well with those derived experimentally.

The model indicates that the ions are accelerated in the radial direction, which strongly suggests a collisionless shock due to hole boring in the underdense plasma from the comparison between the experiment and the computer simulation. The nature of the ion-acceleration mechanism observed here is very revealing. It appears that the focused intensity plays a crucial role in the laser intensity for PW- class laser systems in that the pedestal could have a high enough intensity to generate a long preformed plasma before the main interaction pulse arrives. This effect will also play a role in the lateral transport of electron energy, evident in x-ray $K \alpha$ measurements under identical irradiation conditions [12]. In the context of a cone-focused fast ignitor [24], this would increase the plasma density in the evacuated cone. This should be avoided if at all possible because of the increased stand-off distance and possible reduction of laser energy coupling to the core due to plasma instabilities and the lateral transport of energy, a mechanism which has been presented here even though there is a small possibility of laser propagation in the plasma due to a self-focused channeling. It might be necessary for the next generation of experiments (using the NIF, OMEGA EP, and/or the FIREX lasers) to control the contrast ratio of the heating PW driver, for example by employing an antireflection coated glass plate as a plasma mirror [25].

We thank all the staff of the Central Laser Facility for their help in the execution of this work. This work was supported by a joint United Kingdom Engineering and Physical Sciences Research Council/Ministry of Defense Grant No. GR/R16778. American colleagues acknowledge support from the U.S. Department of Energy Contract No. W-7405Eng-48. Japanese colleagues acknowledge the Japan Society for the Promotion of Science.
[1] M. Tabak et al., Phys. Plasmas 1, 1626 (1994).

[2] V. Yu. Bychenkov et al., JETP 88, 1137 (1999).

[3] P. McKenna et al., Phys. Rev. Lett. 91, 075006 (2003).

[4] D. R. Farley et al., Phys. Rev. Lett. 83, 1982 (1999).

[5] E. L. Clark et al., Phys. Rev. Lett. 85, 1654 (2000).

[6] R. A. Snavely et al., Phys. Rev. Lett. 85, 2945 (2000).

[7] C. Toupin, E. Lefebvre, and G. Bonnaud, Phys. Plasmas 8, 1011 (2001).

[8] A. Maksimchuk, S. Gu, K. Flippo, D. Umstadter, and V. Y. Bychenkov, Phys. Rev. Lett. 84, 4108 (2000).

[9] N. Izumi et al., Phys. Rev. E 65, 036413 (2002).

[10] L. O. Silva et al., Phys. Rev. Lett. 92, 015002 (2004).

[11] L. Gremillet et al., Phys. Rev. Lett. 83, 5015 (1999).

[12] R. B. Stephens et al., Phys. Rev. E (to be published).

[13] P. A. Norreys et al., Plasma Phys. Controlled Fusion 40, 175 (1998)
[14] L. Disdier et al., Phys. Rev. Lett. 82, 1454 (1999).

[15] H. Habara et al., Proc. SPIE 3886, 513 (2000); Phys. Plasmas 10, 3712 (2003).

[16] C. N. Danson et al., Opt. Commun. 103, 392 (1993).

[17] C. N. Danson et al., J. Mod. Opt. 45, 1653 (1998).

[18] G. J. Pert, J. Comput. Phys. 43, 111 (1981).

[19] P. A. Norreys et al., Phys. Plasmas 6, 2150 (1999).

[20] R. G. Hamker, Ph.D. thesis, UCLA (2000); R. A. Fonseca et al., LCNS 2329, III-342 (Springer-Verlag, Heidelberg, 2002).

[21] W. B. Mori, C. Joshi, J. M. Dawson, D. W. Forslund, and J. M. Kindel, Phys. Rev. Lett. 60, 1298 (1988).

[22] S. P. Hatchett et al., Phys. Plasmas 7, 2076 (2000).

[23] A. J. Mackinnon et al., Phys. Rev. Lett. 88, 215006 (2002).

[24] P. A. Norreys et al., Phys. Plasmas 7, 3721 (2000); R. Kodama et al., Nature (London) 412, 798 (2001).

[25] I. Watts et al., Phys. Rev. E 66, 036409 (2002). 